\title{
Model predictive control of sea wave energy converters - Part II: the case of an array of devices
}

\author{
Guang $\mathrm{Li}^{1}$, Mike R. Belmont ${ }^{1}$
}

\begin{abstract}
This paper addresses model predictive control (MPC) of highly-coupled clusters of sea wave energy converters (WECs). Since each WEC is not only a wave absorber but also a wave generator, the motion of each WEC can be affected by the waves generated by its adjacent WECs when they are close to each other. A distributed MPC strategy is developed to maximize the energy output of the whole array and guarantee the safe operation of all the WECs with a reasonable computational load. The system for an array is partitioned into subsystems and each subsystem is controlled by a local MPC controller. The local MPC controllers run cooperatively by transmitting information to each other. Within one sampling period, each MPC controller perform optimizations iteratively so that a global optimization for the whole array can be approximated. The computational burden for the whole array is also distributed to the local controllers. A numerical simulation demonstrates the efficacy of the proposed control strategy. For the WECs operating under constraints explored, it is found that the optimized power output is an increasing function of degree of WEC-WEC coupling. Increases in power of up to $20 \%$ were achieved using realistic ranges of parameters with respect to the uncoupled case.
\end{abstract}

Keywords: wave energy, model predictive control, distributed control

\section{Introduction}

A wave farm usually contains a number of sea wave energy converters (WECs) deployed within a region. The control targets for a farm involve

Email addresses: g.li@exeter.ac.uk (Guang Li), M.R.Belmont@exeter.ac.uk (Mike R. Belmont) 
maximizing energy generation, smoothing the power output for a friendly connection to a grid, reducing hardware cost, and maintaining the safe operation of the devices, etc. Most of the WECs in a farm are normally installed close to each other due to practical considerations, such as space limit, cable deployment, electricity delivery and maintenance. Since each WEC is not only a wave absorber but also a wave generator, the proximity of the WECs means that the motion of each WEC is affected by the waves generated by adjacent ones. This feature clearly complicates both the modelling and the control of the wave farm. Furthermore, the computational cost of control of the whole array can be intractable for real time operations. To address this problem, a hierarchical control system architecture can be employed, in which the overall array is partitioned first into sub-arrays, each of which contains a modest number of neighbouring devices. Thus to a first approximation, we assume strong interactions only occur within sub-arrays, while the sub-array to sub-array interactions are weak. It is then possible to treat each sub-array as a single system from a control perspective that is weakly coupled to similar adjacent ones. If necessary this process can be taken further with the weakly interacting sub-arrays themselves combined into larger scale entities and so on over multiple scales allowing a hierarchical layered control system.

This paper addresses the modelling and control of the modest-sized, highly-coupled arrays of WECs at the lower level of the hierarchical structure. The goal is to maximize the energy output for such an array of devices while guaranteeing their safe operation. Simpler, weak coupling versions, of the same approach can then be applied at each layer of the hierarchical system. The problem of linking the various layers into a multi-scale distributed control system will be the subject of a subsequent publication. In [1], a model predictive control (MPC) strategy is proposed for a single sea WEC to achieve the maximum energy output while maintaining its safe operation through the satisfaction of certain constraints. However, a direct application of the MPC strategy developed in [1] to an array of WECs coupled via wec waves rapidly becomes computationally unrealistic even for the modest sized arrays of interest here. Such direct application of MPC to a whole system is frequently termed centralized MPC. To tackle this problems, a distributed MPC strategy is developed for the control of modest-sized strongly interacting arrays of WECs.

Distributed MPC has been developed in recent years to resolve constrained optimal control problems of large networked systems. The main 
benefits of using distributed MPC are that it can not only distribute the computational burden to each local controller, but also deliver approximately optimal solution for the whole system. There are two branches of distributed MPC strategies: communication-based distributed MPC, e.g.[2], and coordination-based distributed MPC, e.g. [3]. Both types of distributed MPC incorporate the coupling interactions among the subsystems. The main difference between them is that the communication-based distributed MPC optimizes the local objective function separately, while the coordinationbased distributed MPC optimizes the overall objective function so that the optimal solution can converge to that of the centralized MPC. This paper adopts the coordination-based distributed MPC approach, which can guarantee that its optimal solution can approximate that of centralized MPC after a limit number of iterations.

In this paper, a distributed MPC algorithm is tailored for the specific requirements of the control of an array of WECs. The objective function for each individual WEC takes the same form as that adopted for the MPC of a single WEC proposed in [1]. This objective function makes the distributed MPC developed for WEC array different from most existing distributed MPC algorithms, which are mainly employed for tracking or regulation control problems [2]. A detailed model for the application of distributed MPC scheme is developed for an array of up to seven devices; however, for simplicity, numerical simulations are restricted to an array of only two WECs in order to provide ready comparisons against various alternative schemes. Numerical simulations show the following significant results. Firstly, the array controlled by distributed MPC can generate almost the same amount of energy as that controlled by a centralized MPC controller, while the computational burden of each controller does not significantly increase with the number of WECs. Secondly, if each WEC is controlled by a MPC controller without considering the WEC generated waves, reduced energy output and constraint violation can result, which demonstrates the invalidity of the application of the MPC strategy independently for each WEC. Thirdly, if the WECs are assumed to be placed far away from each other so that the influence from the WEC generated waves are insignificant, then output energy of the WECs controlled by local MPC controllers is smaller than that of the WECs when they are close enough so that the generated waves take effect; thus at least for the limited examples explored, when controlling WECs subject to constraints, the economic necessity of placing the WECs close to each other to save space and layout cost, can also result in increased energy output. 
In addition to dynamical models for the WECs and the wave-wave field the MPC scheme also requires some form of deterministic sea wave prediction algorithm (DSWP)[4-10] and far field wave data (a standard multidirectional wave model using Pierson-Moskovitz wave spectra was employed).

The focus here is not on the details of the specific WECs involved, rather on the far wider control issues. Thus for the sake of clarity, relatively simplified generic WEC models are employed, similar to those used in [1]. However given the approach employed, here the incorporation of far more detailed WEC dynamics, particular to a specific technology, is a very straightforward process.

The structure of this paper is as follows. Modeling issues are addressed in Section 2. Three candidate MPC control strategies for the control of an array of WECs are introduced in Section 3. Section 4 focuses on distributed MPC algorithm development. Finally, simulation results are shown in Section 5 to demonstrate the efficacy of the proposed distributed MPC.

\section{Model establishment for an array of WECs}

Consider an array of $m$ WECs, and suppose the motion of any WEC $i$ can be influenced by the waves generated by the remaining $m-1$ WECs. WEC $i$ can be described by a discrete time state space model

$$
\begin{aligned}
x_{i}(k+1) & =A_{i} x_{i}(k)+B_{u i} u_{i}(k)+B_{w i}\left(w_{f, i}(k)+\sum_{l \neq i}^{m-1} w_{i, l}(k)\right) \\
y_{i}(k) & =C_{i} x_{i}(k) \\
z_{i}(k) & =C_{z, i} x_{i}(k)
\end{aligned}
$$

Here $y_{i}, x_{i}$ and $u_{i}$ are the heave motion, state variable and control signal respectively. $z_{i}$ represents a constrained state variable (constrained for safety reasons), $w_{f, i}$ is time derivative of the vertical displacement of the far field (external) wave as propagated to WEC $i$, which is required to be predicted by the DSWP algorithm. $w_{i, l}$ represents the time derivative of the vertical displacement of the WEC wave at WEC $i$ that was generated by WEC $l$. The quantity $w_{i, l}$ is dependent on two factors: a) the locations of the WECs $i$ and $l$, and $\mathrm{b}$ ) the heave motion of WEC $l$. There are two approaches to obtaining $w_{i, l}$. One is via a first-principle based fluid mechanical model, in general, including nonlinearities. The other more straightforward, and 
perhaps more practical method, is to ignore the nonlinearities between $w_{i, l}$ and $y_{l}$, so that the dynamics can be derived using standard linear system identification methods based on experimental data. In the latter case, this relation can be expressed in the frequency domain

$$
\hat{W}_{i, l}=\hat{H}_{i, l} \hat{Y}_{l},
$$

where $\hat{H}_{i, l}$ represents the frequency response function between the internal wave $\hat{W}_{i, l}$ and the motion of WEC $l, \hat{Y}_{l}$, which are the Fourier transforms of $w_{i}$, and $y_{l}$ respectively.

For numerical simulation, we establish the fluid mechanical model as in [11]

$$
\hat{H}_{i, l}=j \omega \sqrt{\frac{\pi|\omega|^{4}}{8 g^{3}}} a^{2} \frac{1}{\sqrt{d_{i, l}}} e^{-j\left\{\frac{\omega^{2}}{g} d_{i, l}+\frac{3 \pi}{4}\right\}}
$$

Here $d_{i, l}$ is the distance between the locations of the WEC $l$ generating wave and the WEC $i$ under consideration; $a$ is the diameter of the float; $g=9.81$ $\mathrm{m} / \mathrm{s}^{2}$ is the gravitational constant. It is assumed for the dynamics model (3) that $k(\omega) a \ll 1$, where $k(\omega) \approx \frac{\omega^{2}}{g}$ is the wave number for deep water. Note that care should be taken for equation (3) to ensure the correct symmetry properties such that the appropriate Fourier inverses stay real.

For $\omega>0,(3)$ can be equivalently written as

$$
\hat{H}_{i, l}=A(\omega) e^{-j \phi(\omega)}
$$

with

$$
\begin{aligned}
& A(\omega)=\omega^{3} a^{2} \sqrt{\frac{\pi}{8 g^{3} d_{i, l}}} \\
& \phi(\omega)=\frac{d_{i, l} \omega^{2}}{g}+\frac{\pi}{4}
\end{aligned}
$$

Using the frequency-domain identification, a rational proper transfer function $H_{i, j}$ can be derived to approximate (4). A numerical example is given in the numerical simulation shown later in this paper.

Suppose the discrete-time state-space model of $H_{i, l}$ is

$$
\begin{aligned}
\xi_{i, l}(k+1) & =E_{i, l} \xi_{i, l}(k)+F_{i, l} y_{l}(k) \\
w_{i, l}(k) & =M_{i, l} \xi_{i, l}(k)
\end{aligned}
$$




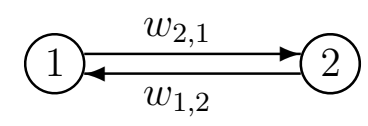

Figure 1: The interaction between 2 WECs

where $\xi_{i, l} \in \mathbb{R}^{n_{w i, l}}$ is the state of the WEC wave model. In the above approach the WEC-WEC wave coupling allows for incorporation of the gravity wave dispersion relationship over the frequency range that significantly affects the relevant WECs to whatever level of approximation is required.

Based on (1) and (7), the process of developing an array model is initially demonstrated for the simplest possible case of two interacting WECs. A more complicated example for an array of 7 WECs is presented in Appendix A.

\section{Example 1 - an array of 2 WECs}

By combining the dynamics of the two WECs expressed by equation (1a) with $i=1,2, m=2$ and the wave interactions expressed by (7) together, we can derive an augmented WEC model:

$$
\begin{aligned}
\mathbf{x}_{i}(k+1) & =\mathbf{A}_{i} \mathbf{x}_{i}(k)+\mathbf{B}_{u, i} u_{i}(k)+\mathbf{B}_{w, i} w_{f, i}(k)+\mathbf{F}_{i, l} \mathbf{x}_{j}(k) \\
\mathbf{y}_{i}(k) & =\mathbf{C}_{i} \mathbf{x}_{i}(k) \\
\mathbf{z}_{i}(k) & =\mathbf{C}_{z, i} \mathbf{x}_{i}(k)
\end{aligned}
$$

with

$$
\begin{aligned}
& \mathbf{A}_{i}=\left[\begin{array}{cc}
A_{i} & B_{w, i} M_{i, l} \\
0 & E_{i, l}
\end{array}\right], \quad \mathbf{B}_{u, i}=\left[\begin{array}{c}
B_{u i} \\
0
\end{array}\right], \quad \mathbf{B}_{w, i}=\left[\begin{array}{c}
B_{w i} \\
0
\end{array}\right], \\
& \mathbf{F}_{i, l}=\left[\begin{array}{cc}
0 & 0 \\
F_{i, l} C_{l} & 0
\end{array}\right], \quad \mathbf{C}_{i}=\left[\begin{array}{ll}
C & 0
\end{array}\right], \quad \mathbf{C}_{z, i}=\left[\begin{array}{ll}
C_{z} & 0
\end{array}\right]
\end{aligned}
$$

Here if $i=1$, then $l=2$ and if $i=2$, then $l=1 ; \mathbf{x}_{i}:=\left[x_{i}^{T}(k), \xi_{i, l}^{T}(k)\right]^{T}$ denotes the augmented state of WEC $i$. We call (8) partitioned model, which combines the state variables of WEC $i$ and WEC $l$. We can also write the 
model of the two WECs together as

$$
\begin{aligned}
{\left[\begin{array}{l}
\mathbf{x}_{1}(k+1) \\
\mathbf{x}_{2}(k+1)
\end{array}\right]=} & {\left[\begin{array}{cc}
\mathbf{A}_{1} & \mathbf{F}_{1,2} \\
\mathbf{F}_{2,1} & \mathbf{A}_{2}
\end{array}\right]\left[\begin{array}{l}
\mathbf{x}_{1}(k) \\
\mathbf{x}_{2}(k)
\end{array}\right]+\left[\begin{array}{cc}
\mathbf{B}_{u, 1} & \mathbf{0} \\
\mathbf{0} & \mathbf{B}_{u, 2}
\end{array}\right]\left[\begin{array}{l}
u_{1}(k) \\
u_{2}(k)
\end{array}\right] } \\
& +\left[\begin{array}{cc}
\mathbf{B}_{w, 1} & \mathbf{0} \\
\mathbf{0} & \mathbf{B}_{w, 2}
\end{array}\right]\left[\begin{array}{l}
w_{f, 1}(k) \\
w_{f, 2}(k)
\end{array}\right]
\end{aligned}
$$

which is called a centralized model.

More generically, if there are $m$ WECs in an array, the partitioned model of WEC $i$ is

$$
\begin{aligned}
\mathbf{x}_{i}(k+1) & =\mathbf{A}_{i} \mathbf{x}_{i}(k)+\mathbf{B}_{u, i} u_{i}(k)+\mathbf{B}_{w, i} w_{f, i}(k)+\sum_{l \neq i}^{m} \mathbf{F}_{i, l} \mathbf{x}_{l}(k) \\
\mathbf{y}_{i}(k) & =\mathbf{C}_{i} \mathbf{x}_{i}(k) \\
\mathbf{z}_{i}(k) & =\mathbf{C}_{z, i} \mathbf{x}_{i}(k)
\end{aligned}
$$

with $i=1, \ldots, m$. The generic centralized model for $m$ WECs is

$$
\mathbf{x}(k+1)=\mathbf{A} \mathbf{x}(k)+\mathbf{B}_{u} \mathbf{u}(k)+\mathbf{B}_{w} \mathbf{w}_{f}(k)
$$

with $\mathbf{x}=\left[\mathbf{x}_{1}^{T}, \ldots, \mathbf{x}_{m}^{T}\right], \mathbf{u}=\left[u_{1}^{T}, \ldots, u_{m}^{T}\right], w_{f}=\left[w_{f, 1}^{T}, \ldots, w_{f, m}^{T}\right]$, and

$$
\begin{gathered}
\mathbf{A}=\left[\begin{array}{ccccc}
\mathbf{A}_{1} & \mathbf{F}_{1,2} & \mathbf{F}_{1,3} & \ldots & \mathbf{F}_{1, m} \\
\mathbf{F}_{2,1} & \mathbf{A}_{2} & \mathbf{F}_{2,3} & \ldots & \mathbf{F}_{2, m} \\
\mathbf{F}_{3,1} & \mathbf{F}_{3,3} & \mathbf{A}_{3} & \ldots & \mathbf{F}_{3, m} \\
\vdots & \vdots & \vdots & \ddots & \vdots \\
\mathbf{F}_{m, 1} & \mathbf{F}_{m, 2} & \mathbf{F}_{m, 3} & \cdots & \mathbf{A}_{m}
\end{array}\right] \\
\left.\mathbf{B}_{u, m}\right), \mathbf{B}_{w}=\operatorname{blkdiag}\left(\mathbf{B}_{w, 1}, \cdots, \mathbf{B}_{w, m}\right) .
\end{gathered}
$$

\section{MPC control strategies for an array of WECs}

In all the three control strategies introduced in this section, the trajectory of input slew rate with a horizon $M$ is used as the optimization variable. The output horizon is $N$. To guarantee the safe operation of the WEC $i$ and realistic control inputs, the following constraints are required to be satisfied

$$
\begin{aligned}
& \left|z_{i}(j)\right| \leq z_{i, \max } \text { for } j=0,1, \ldots, N \\
& \left|u_{i}(j)\right| \leq u_{i, \max } \text { for } j=0,1, \ldots, N \\
& \left|\Delta u_{i}(j)\right| \leq \Delta u_{i, \max } \text { for } j=0,1, \ldots, M
\end{aligned}
$$

where $z_{i, \max }, u_{i, \max }$ and $\Delta u_{i, \max }$ are limits for the absolute values of the constrained state, the input magnitude and input slew rate respectively. 


\subsection{Decentralized $M P C$}

The first case considered deals with the situation where the WECs are deployed far apart so that the WEC waves are too weak to influence the adjacent WECs. This may seem a trivial example, and equivalent to the single WEC control problem; however, it is valuable as it shares all of the development of the more complex case of the interacting array and hence provides a useful introduction to the methodology.

Model (1a) for WEC $i$ is thus reduced to

$$
x_{i}(k+1)=A_{i} x_{i}(k)+B_{u i} u_{i}(k)+B_{w i} w_{f, i}(k)
$$

For this case, a local MPC controller developed in [1] can be employed directly for each WEC. As shown in [1], the objective function for WEC $i$ is chosen to be

$$
J_{i}=\sum_{j=0}^{N}\left[y_{i}(k+j) u_{i}(k+j)+r_{i} u_{i}^{2}(k+j)+q_{i} z_{i}^{2}(k+j)\right]
$$

where the term $y_{i}(k+j) u_{i}(k+j)$ represents the power generated by WEC $i$; the term $r_{i} u_{i}^{2}(k+j)$ is used to penalize the power consumed by control input; the term $q_{i} z_{i}^{2}(k+j)$ is used to penalize the magnitude of the constrained variable; in general, the positive scalars $r_{i}$ and $q_{i}$ are used to tradeoff the energy output, energy consumed by actuator and state constraint. Based on the objective function (14) and the model of WEC $i$, a local MPC controller can be designed to control each WEC separately by resolving a constrained optimization problem online at each sampling instant:

$$
\begin{aligned}
& \min J_{i} \\
& \text { subject to (13) and (12) }
\end{aligned}
$$

We call this control strategy decentralized $M P C$.

\subsection{Centralized $M P C$}

A direct approach to take into account the interactions of WECs of an array is to use the centralized model (9) and adopt a central MPC controller. The objective function for an array of $m$ WECs is

$$
J=\sum_{i=1}^{m} w_{i} J_{i}
$$


with $w_{i}>0$ and $\sum_{i=1}^{m} w_{i}=1$. The central MPC controller aims to control the whole array together by resolving a constrained optimization problem online at each sampling instant:

$$
\begin{aligned}
& \min J \\
& \text { subject to (11) and (12) with } i=1, \ldots, m .
\end{aligned}
$$

Such centralized MPC guarantees the Pareto optimal solution of (17). However the main problem can be from the computational burden, since the dimension of the array's model can increase dramatically with the number of WECs.

\subsection{Distributed $M P C$}

The third scheme explored is distributed $M P C$. For an array of $m$ WECs, the whole system is divided into $m$ subsystems, each of which is controlled by its own MPC controller. This is described as the partitioned model (10), The computational burden caused by a large dimensional optimization problem (17) for the whole array is thus distributed to each local MPC controller. Rather than running independently as the decentralized MPC, these MPC controllers are coordinated and run iteratively to approximate the optimal solution achieved by centralized MPC. Each local MPC controller resolves a constrained optimization problem iteratively online within each sampling period:

$$
\begin{aligned}
& \min J \\
& \text { subject to (10) and (12) with } i=1, \ldots, m .
\end{aligned}
$$

In the next section, this distributed MPC is developed for the control of an array of WECs.

\section{Distributed MPC algorithm development for an array of WECs}

In this section we develop distributed MPC algorithm for an array of $m$ WECs. It is necessary to first reformulate the objective function in the optimization problem (18) into a quadratic form.

If the actuator's slew rate needs to be limited, the input slew rate has to be used as the optimization variable. We assume $M$ control moves of $\Delta u(k+j):=u(k+j)-u(k+j-1)$ with $j=1, \ldots, M$ and a prediction 
horizon of $N$ steps for the output, with $M \leq N$. Then we have the following relations

$$
\begin{gathered}
u(k+i)=u(k-1)+\sum_{j=0}^{i} \Delta u(k+j-1) \text { with } i=0,1, \ldots, M, \\
u(k+M)=u(k+M+1)=\cdots=u(k+N) .
\end{gathered}
$$

Define the trajectories for the state, output, constrained state, input slew rate, input and predicted external wave for WEC $i$

$$
\begin{aligned}
\overline{\mathbf{x}}_{i} & =\left[\mathbf{x}_{i}(k), \mathbf{x}_{i}(k+1), \ldots, \mathbf{x}_{i}(k+N)\right]^{T} \\
\overline{\mathbf{y}}_{i} & =\left[\mathbf{y}_{i}(k), \mathbf{y}_{i}(k+1), \ldots, \mathbf{y}_{i}(k+N)\right]^{T} \\
\overline{\mathbf{z}}_{i} & =\left[\mathbf{z}_{i}(k), \mathbf{z}_{i}(k+1), \ldots, \mathbf{z}_{i}(k+N)\right]^{T} \\
\Delta \overline{\mathbf{u}}_{\mathbf{i}} & =\left[\Delta u_{i}(k), \Delta u_{i}(k+1), \ldots, \Delta u_{i}(k+M-1)\right]^{T} \\
\overline{\mathbf{u}}_{\mathbf{i}} & =\left[u_{i}(k), u_{i}(k+1), \ldots, u_{i}(k+N-1)\right]^{T} \\
\overline{\mathbf{w}}_{f, i} & =\left[w_{f, i}(k), w_{f, i}(k+1), \ldots, w_{f, i}(k+N-1)\right]^{T}
\end{aligned}
$$

Equations (19a) and (19b) can be expressed by a matrix equation as

$$
\overline{\mathbf{u}}_{i}=T_{u} u_{i}(k-1)+T_{\Delta} \Delta \overline{\mathbf{u}}_{i}
$$

where

$$
T_{u}=\left[\begin{array}{c}
1 \\
1 \\
\vdots \\
1 \\
\vdots \\
1
\end{array}\right] \quad T_{\Delta}=\left[\begin{array}{cccc}
1 & 0 & 0 & 0 \\
1 & 1 & 0 & 0 \\
\vdots & \vdots & \ddots & \\
1 & 1 & \cdots & 1 \\
\vdots & \vdots & \cdots & \vdots \\
1 & 1 & \cdots & 1
\end{array}\right]
$$

with $T_{u} \in \mathbb{R}^{(N+1) \times 1}$ and $T_{\Delta} \in \mathbb{R}^{(N+1) \times M}$. As shown in Appendix B, the following relations hold

$$
\begin{gathered}
\overline{\mathbf{y}}=\boldsymbol{\Theta}_{u} \mathbf{u}(k-1)+\boldsymbol{\Psi} \Delta \overline{\mathbf{u}}+\boldsymbol{\Theta}_{x} \mathbf{x}+\boldsymbol{\Theta}_{w} \overline{\mathbf{w}} \\
\overline{\mathbf{z}}=\boldsymbol{\Theta}_{z, u} \mathbf{u}(k-1)+\boldsymbol{\Psi}_{z} \Delta \overline{\mathbf{u}}+\boldsymbol{\Theta}_{z, x} \mathbf{X}+\boldsymbol{\Theta}_{z, w} \overline{\mathbf{w}}
\end{gathered}
$$


and

$$
\begin{aligned}
& \overline{\mathbf{y}}_{i}=\boldsymbol{\Psi}_{i, i} \Delta \overline{\mathbf{u}}_{i}+\sum_{j \neq i}^{m} \boldsymbol{\Psi}_{i, j} \Delta \overline{\mathbf{u}}_{j}+\boldsymbol{\Theta}_{x, i} \mathbf{x}+\boldsymbol{\Theta}_{w, i} \overline{\mathbf{w}}+\boldsymbol{\Theta}_{u, i} \mathbf{u}(k-1) \\
& \overline{\mathbf{z}}_{i}=\boldsymbol{\Psi}_{z, i, i} \Delta \overline{\mathbf{u}}_{i}+\sum_{j \neq i}^{m} \boldsymbol{\Psi}_{z, i, j} \Delta \overline{\mathbf{u}}_{j}+\boldsymbol{\Theta}_{z, x, i} \mathbf{x}+\boldsymbol{\Theta}_{z, w, i} \overline{\mathbf{w}}+\boldsymbol{\Theta}_{z, u, i} \mathbf{u}(k-1)
\end{aligned}
$$

where $\boldsymbol{\Psi}_{i, j}$ and $\boldsymbol{\Psi}_{z, i, j}$ are row vectors derived from the row $i$ and the columns from $(i-1) \times m+1$ to $i \times m$ of $\boldsymbol{\Psi}$ and $\boldsymbol{\Psi}_{z}$ respectively; $\boldsymbol{\Theta}_{z, x, i}, \boldsymbol{\Theta}_{z, w, i}$ and $\boldsymbol{\Theta}_{z, u, i}$ are the row $i$ of $\boldsymbol{\Theta}_{z, x}, \boldsymbol{\Theta}_{z, w}$ and $\boldsymbol{\Theta}_{z, u}$ respectively; the concatenated initial state vectors, input magnitudes and predicted external waves for $m$ WECs are

$$
\mathbf{u}(k-1)=\left[\begin{array}{c}
u_{1}(k-1) \\
u_{2}(k-1) \\
\vdots \\
u_{m}(k-1)
\end{array}\right], \quad \overline{\mathbf{w}}=\left[\begin{array}{c}
\overline{\mathbf{w}}_{f, 1} \\
\overline{\mathbf{w}}_{f, 2} \\
\vdots \\
\overline{\mathbf{w}}_{f, m}
\end{array}\right], \quad \mathbf{x}=\left[\begin{array}{c}
\mathbf{x}_{1}(k) \\
\mathbf{x}_{2}(k) \\
\vdots \\
\mathbf{x}_{m}(k)
\end{array}\right]
$$

Using these relations, we can derive the objective function for WEC $i$ in a quadratic form with $\Delta \overline{\mathbf{u}}_{i}$ as the optimization variable:

$$
J\left(\Delta \overline{\mathbf{u}}_{i}, p\right)=\frac{1}{2} \Delta \overline{\mathbf{u}}_{i}^{T} \mathbf{H}_{i} \Delta \overline{\mathbf{u}}_{i}+\Delta \overline{\mathbf{u}}_{i}^{T} \mathbf{F}_{i}
$$


where

$$
\begin{aligned}
\mathbf{H}_{i}= & T_{\Delta}^{T} \boldsymbol{\Psi}_{i, i}+\boldsymbol{\Psi}_{i, i}^{T} T_{\Delta}+2 T_{\Delta}^{T} R T_{\Delta}+2 \sum_{j=1}^{m} \boldsymbol{\Psi}_{z, j, i}^{T} Q \boldsymbol{\Psi}_{z, j, i} \\
\mathbf{F}_{i}= & \sum_{t \neq i}^{m}\left[T_{\Delta}^{T} \boldsymbol{\Psi}_{i, t}+2 \sum_{j=1}^{m} \boldsymbol{\Psi}_{z, j, i}^{T} Q \boldsymbol{\Psi}_{z, j, t}\right] \Delta \overline{\mathbf{u}}_{t}^{p-1} \\
& +\left[T_{\Delta}^{T} \boldsymbol{\Theta}_{x, i}+2 \sum_{j=1}^{m} \boldsymbol{\Psi}_{z, j, i}^{T} Q \boldsymbol{\Theta}_{z, x, j}\right] \mathbf{x} \\
& +\left[T_{\Delta}^{T} \boldsymbol{\Theta}_{w, i}+2 \sum_{j=1}^{m} \boldsymbol{\Psi}_{z, j, i}^{T} Q \boldsymbol{\Theta}_{z, w, j}\right] \overline{\mathbf{w}} \\
& +\left[T_{\Delta}^{T} \boldsymbol{\Theta}_{u, i}+2 \sum_{j=1}^{m} \boldsymbol{\Psi}_{z, j, i}^{T} Q \boldsymbol{\Theta}_{z, u, j}\right] \mathbf{u}(k-1) \\
& +2 T_{\Delta}^{T} R T_{u} u_{i}(k-1)+\sum_{j=1}^{m} \boldsymbol{\Psi}_{j, i}^{T} T_{u} u_{j}(k-1)
\end{aligned}
$$

Here $\boldsymbol{\Delta} \overline{\mathbf{u}}_{j}^{p-1}$ denotes the optimal solution of WEC $j$ derived at the last iteration, $p-1$. The overall optimization of the whole array is achieved by resolving separate optimization problem of each WEC iteratively.

Moreover, the constraints (12) can be represented by

$$
\begin{aligned}
-\Delta U_{\text {max }} & \preceq \Delta \overline{\mathbf{u}}_{i} \preceq \Delta U_{\text {max }} \\
-U_{\text {max }} & \preceq \overline{\mathbf{u}}_{i} \preceq U_{\text {max }} \\
-Z_{\text {max }} & \preceq \overline{\mathbf{z}}_{i} \preceq Z_{\text {max }}
\end{aligned}
$$

where $\Delta U_{\max }:=\left[\Delta u_{\max }, \cdots, \Delta u_{\max }\right]^{T}, U_{\max }:=\left[u_{\max }, \cdots, u_{\max }\right]^{T}, Z_{\max }:=$ $\left[z_{\max }, \cdots, z_{\max }\right]^{T}$, and $\preceq$ denotes component-wise inequality. For WEC $i$ these constraints at iteration $p$ can be written in a compact form as

$$
\mathcal{A}_{i} \Delta \overline{\mathbf{u}}_{i} \preceq \mathcal{B}_{i}
$$


with

$$
\begin{aligned}
\mathcal{A}_{i}= & {\left[\begin{array}{c}
I \\
-I \\
T_{\Delta} \\
-T_{\Delta} \\
\boldsymbol{\Psi}_{z, i, i} \\
-\boldsymbol{\Psi}_{z, i, i}
\end{array}\right] } \\
\mathcal{B}_{i}= & {\left[\begin{array}{c}
\Delta U_{\max } \\
\Delta U_{\max } \\
Z_{\max }-\sum_{j \neq i}^{m} \boldsymbol{\Psi}_{z, i, j} \Delta \overline{\mathbf{u}}_{j}^{p-1}-\boldsymbol{\Theta}_{z, x, i} \mathbf{x}-\Theta_{z, w, i} \overline{\mathbf{w}}-\boldsymbol{\Theta}_{z, u, i} \mathbf{u}(k-1) \\
U_{\max }+\sum_{j \neq i}^{m} \mathbf{\Psi}_{z, i, j} \Delta \overline{\mathbf{u}}_{j}^{p-1}+\boldsymbol{\Theta}_{z, x, i} \mathbf{x}+\boldsymbol{\Theta}_{z, w, i} \mathbf{\mathbf { w }}+\boldsymbol{\Theta}_{z, u, i} \mathbf{u}(k-1)
\end{array}\right] }
\end{aligned}
$$

For WEC $i$, the optimization resolved at iteration $p$ is

$$
\mathcal{P}_{i}^{p}:=\min _{\Delta \overline{\mathbf{u}}_{i}} \sum_{i=1}^{m} J\left(\Delta \overline{\mathbf{u}}_{i}, p\right)
$$

s.t. $(26)$

for $i=1, \ldots, m$. The optimal slew rate trajectory at iteration $p$ can be derived by

$$
\Delta \overline{\mathbf{u}}_{i}^{p(*)}=\arg \min \mathcal{P}_{i}^{p}
$$

Based on the above development, we have the distributed MPC algorithm as shown in Algorithm 1. Algorithm 1 can guarantee that the optimal solution of the distributed MPC converges to that of the centralized MPC for large $p$. This can be shown by following the similar proof procedure in [3]. The bock diagram of the distributed MPC for an array of $m$ WECs is illustrated in Fig. 4. Here $K_{i}$ with $i=1, \ldots, m$ are the local controllers and observers, which run the distributed MPC Algorithm. $P_{i}$ with $i=1, \ldots, m$ stand for the DSWP prediction algorithm for WEC $i$. At each sampling instant, the state of each WEC is estimated by an observer. The estimated states and the predicted wave $\bar{w}_{f, i}$ are fed into the local controller $K_{i}$, which performs one iteration of optimization. The optimal solutions and the estimated states of each WEC are shared by other ones through the "Communication and Coordination" block. Suppose $p_{\max }$ is an upper 

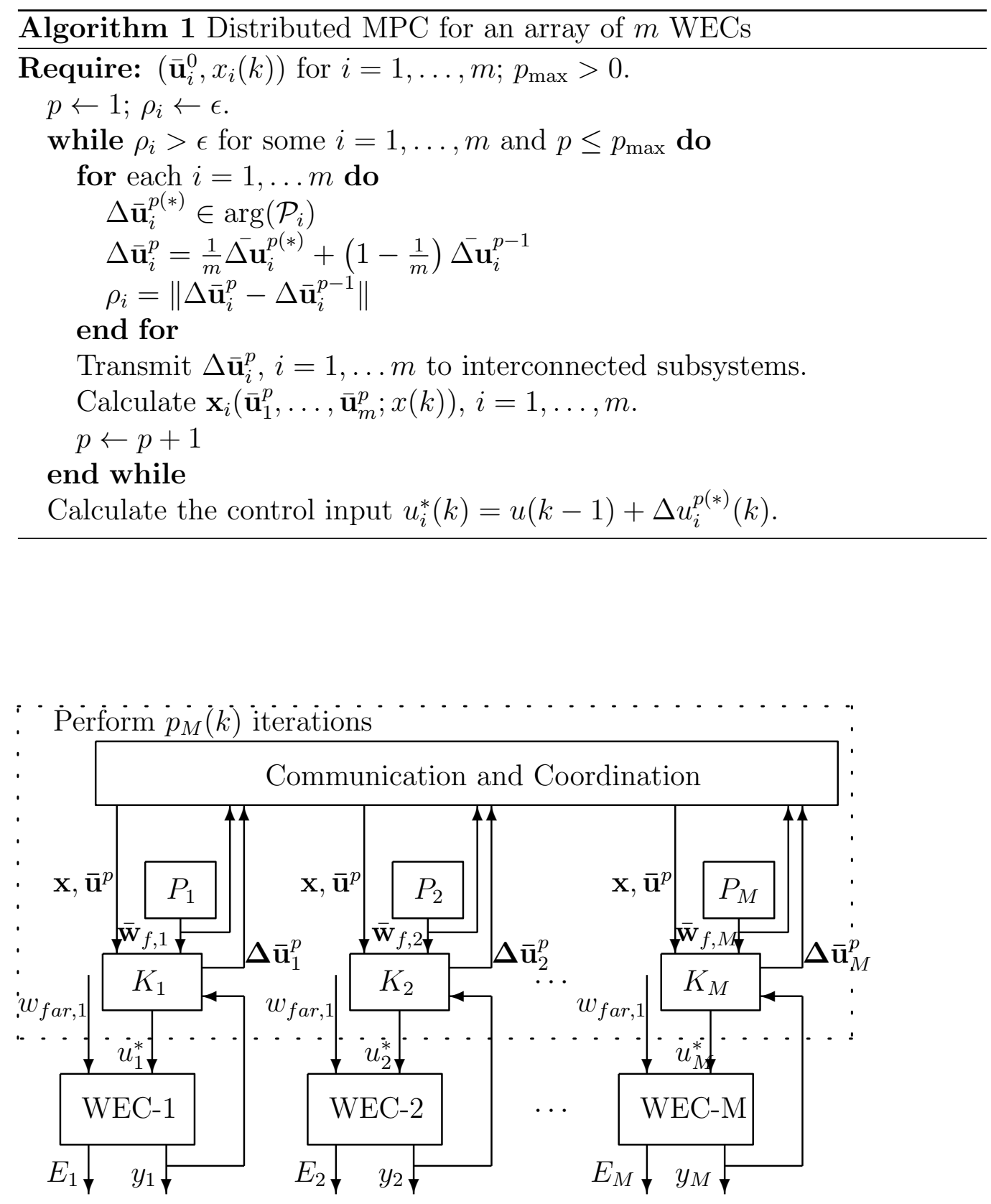
limit for the number of iterations. At sampling instant $k, p_{i}(k) \leq p_{\max }$ iterations is performed, after which the first element of the resulting optimal slew rate sequence, $\Delta u_{i}^{*}(k)$, is derived and the control input for WEC $i$ is $u_{i}^{*}(k)=u(k-1)+\Delta u_{i}^{*}(k)$ for WEC $i$.

\section{Simulation}

In this section, numerical simulations for an array of two WECs are demonstrated to compare the performances of the three MPC control strategies. The two WECs are assumed identical and have the similar dynamic model as the example in $[12,13]$. Thus, $A_{1}=A_{2}, B_{1}=B_{2}, C_{1}=C_{2}$, $B_{w, 1}=B_{w, 2}$ and $B_{u 1}=B_{u, 2}$ in the model (1) with $i=1,2$. The damping ration is frequency dependent. The cross section area and the mass of the float are assumed bigger than the original values, so that the coupling effect between the WECs are significant; these values used in the simulations are summarized in Table 1. Note that the constraints on the input magnitude, input slew rate are $3 \times 10^{5} \mathrm{~N}$ and $4 \times 10^{4} \mathrm{~N}$ respectively. The constrained state represents the difference between the heaves of the sea and float. This state constraint is $1.2 \mathrm{~m}$.

The far waves are created using a standard multi-directional wave model based on Pierson-Moskovitz wave spectra.The distance between two WECs are assumed to be $90 \mathrm{~m}$. The heaves and the first derivatives of the heaves of the two wave sequences are correlated. The root mean square (rms) values of the two wave sequences are $3 \mathrm{~m}$.

The dynamics of the wave generated by WECs with different radius can be derived from (3) and further approximated using frequency-domain system identification MATLAB ${ }^{\mathrm{TM}}$ toolbox. As an example, when the distance between the two WECs is $d=90 \mathrm{~m}$ and the radius of the float is $10 \mathrm{~m}$. The approximated transfer function is

$$
H_{1,2}(s)=H_{2,1}(s)=\frac{0.2893 s^{4}-0.1893 s^{3}+0.3192 s^{2}-0.09092 s+0.04864}{s^{5}+1.256 s^{4}+2.015 s^{3}+1.677 s^{2}+0.9051 s+0.4606}
$$

For comparison, three MPC control strategies are implemented. The decentralized MPC is also implemented for the case when the two WECs are assumed to be decoupled through the waves generated by themselves.

Distributed MPC (DSMPC) Two distributed MPC controller resolves the optimization (27) coordinately and iteratively during one sampling 
period. The iteration number is set to $p_{\max }=2$ for the distributed MPC. The performance can be further improved with an increased value of $p_{\max }$, and it eventually converges to the performance of centralized MPC.

Centralized MPC (CMPC) A centralized MPC controller resolves a constrained optimization (17) within one sampling period.

Decentralized MPC (DCMPC) Two local MPC controllers resolve constrained optimization (15) independently.

Decentralized MPC - decoupled case (DCMPCd) Two local MPC controllers resolve constrained optimization (15) independently. The two WECs are assumed to be placed far away from each other so that the generated waves are negligible.

In all the simulations, the sampling time is $0.02 \mathrm{~s}$, prediction horizons are $N=60$ and $M=30$. The weights are tuned properly for each scenario to achieve the maximum energy output. We first demonstrate the results when the radius of the WECs is $r=10 \mathrm{~m}$, the float mass (including added mass) is $m=2.8 \times 10^{5} \mathrm{~kg}$, and the the distance between them is $d=90 \mathrm{~m}$.

Fig. 2 shows that the control signals in the four cases all satisfy the input constraints.

Fig. 3 shows the values of the constrained variable, i.e. the vertical displacement difference between the water level and the mid-point of the float. The state constraints are also satisfied for all the cases; but bigger values of $q$ and $r$ are needed for the decentralized MPC when WEC waves are present.

Fig. 4 demonstrates the energy outputs of the two WECs in the four cases. It is obvious that the energy output of the WECs controlled by the distributed MPC is very close to that of WECs controlled by centralized MPC. The WECs controlled by the decentralized MPC generates less energy than the WECs controlled by distributed MPC and centralized MPC; although this energy reduction is not significant, the decentralized MPC normally needs bigger values of $q$ and $r$ to guarantee the satisfaction of constraints for a longer time of simulation due to the ignorance of the generated waves, which leads to much less energy. However, when the two WECs are not coupled by the waves generated by themselves, the two WECs controlled by the decentralized MPC is the best option. Fig. 4 shows that in this case the least 
energy is generated; this result confirms that the WEC generated waves help increase energy output and the deployment of WECs can therefore influence energy generation.

We remark that a fundamental theory in the literature [14] is that the maximum power captured by a WEC is half of the incident wave power. This theory is for a single WEC when no constraints are present, and thus it does not apply for cases in this paper. The significance of our finding is that the when WECS are controlled subject to constraints in the presence of the coupling between the WECs, by virtue of WEC waves, the combined two WEC system can be capable of being controlled to absorb more energy than two uncoupled WECs. This increased energy output can be accounted for by the fact that in the presence of constraints the presence of the WEC waves changes the dynamics of the array which contains the dynamics from both WECs and WEC generated waves.

We further conduct simulations with different values of radius and the masses, but the same distance $d=90 \mathrm{~m}$ between the two WECs. For each case, four MPC strategies are compared. The energy outputs are shown in Table 1. We can see that for each case, the generated energies match the trend shown in Fig. 4, i.e. CMPC $>$ DSMPC $>$ DCMPC $>$ DCMPCd; the energy increase rate for the WECs controlled by DSMPC in the coupled case over the DCMPCd in the decoupled case decreases with the size of the WEC. This is because when the distance $d$ is unchanged, the smaller size of WEC generate waves with less energy, which makes the coupling weaker. This reveals that the dimensions of the WECs and the distances between them are important factors to be considered during WEC deployment.

Finally, it is noted that although the reduction in computational burden for the distributed MPC compared with the centralized MPC is not large for this simple array of two WECs, this reduction of computational burden becomes obviously significant if the number of WECs increases, specifically if $m>p_{\max }$.

\section{Conclusion}

A decentralized MPC control strategy is developed for the control of a moderate-sized and highly-coupled array of WECs. The model establishment for an array of WECs involving the waves generated by themselves is demonstrated. This decentralized MPC can explicitly take into account the WEC waves. The WECs controlled by this distributed MPC can generate 


\begin{tabular}{cc|ccccc}
\hline $\begin{array}{c}\text { radius } \\
(\mathrm{m})\end{array}$ & $\begin{array}{c}\text { mass } \\
\left(\times 10^{5} \mathrm{~kg}\right)\end{array}$ & $\begin{array}{c}\text { DSMPC } \\
\left(\times 10^{7} \mathrm{~J}\right)\end{array}$ & $\begin{array}{c}\text { CMPC } \\
\left(\times 10^{7} \mathrm{~J}\right)\end{array}$ & $\begin{array}{c}\text { DCMPC } \\
\left(\times 10^{7} \mathrm{~J}\right)\end{array}$ & $\begin{array}{c}\text { DCMPCd } \\
\left(\times 10^{7} \mathrm{~J}\right)\end{array}$ & $\begin{array}{c}\mathrm{r} \% \\
-\end{array}$ \\
\hline 10 & 2.8 & 9.7808 & 9.7941 & 9.5156 & 8.2786 & $18.15 \%$ \\
9 & 2.3 & 9.3054 & 9.3248 & 9.1067 & 9.1067 & $13.72 \%$ \\
8 & 1.9 & 8.9054 & 8.9241 & 8.6742 & 8.6742 & $9.74 \%$ \\
\hline
\end{tabular}

Table 1: The energy outputs of the two-WEC array in different scenarios. $r \%=($ DSMPCDCMPCd)/DCMPCd \% is the energy improvement of DSMPC over DCMPCd.

almost the same amount of energy with the case when a centralized MPC is used, while the computational burden is distributed to each local controller, which makes it more practical for implementation on an array of WECs. The simulation confirms the efficacy of using the proposed distributed MPC. It also demonstrates that the generated waves can help improve energy output; this indicates that deployment of WECs can significantly influence the WECs' performances. A hierarchical approach is outlined for the optimal near control of a whole farm using a hierarchical control system structure exploiting the techniques developed. A study of a wave farm operation under such hierarchical control will be the focus of a subsequent report.

\section{Appendix A. Example 2 - modeling for an array of 7 WECs}

Consider an array of 7 WECs, numbered from $i=1$ to 7 , as shown in Fig. A.5. We assume all the WECs are identical and can be represented by the same dynamic model. Every three adjacent WECs are on the vertices of an equilateral triangle with each side a length $d$. There are 3 types of dynamic relations among the WECs through internal waves. Let us take WEC 1 as an example:

Type I Since WEC 1 has the same distance $d$ to WECs 2, 3 and 4, we assume $H_{1,2}=H_{1,3}=H_{1,4}:=H_{a}$. The internal wave acting on WEC 1 is derived from the superposition of the waves generated by WECs 2, 3 and 4 such that $w_{1, a}:=w_{1,2}+w_{1,3}+w_{1,4}$. Then we have the relation

$$
W_{1, a}(j \omega)=H_{a}(j \omega)\left(Y_{2}(j \omega)+Y_{3}(j \omega)+Y_{4}(j \omega)\right)
$$

The state space realization of $H_{a}(j \omega)$ is

$$
\begin{aligned}
\xi_{1, a}(k+1) & =E_{a} \xi_{1, a}(k)+F_{a}\left(y_{2}(k)+y_{3}(k)+y_{4}(k)\right) \\
w_{1, a}(k) & =M_{a} \xi_{1, a}(k)
\end{aligned}
$$




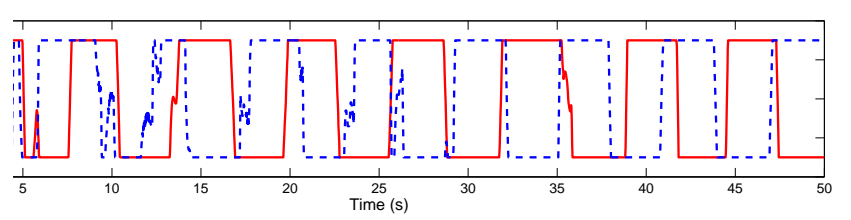

(a) Distributed MPC

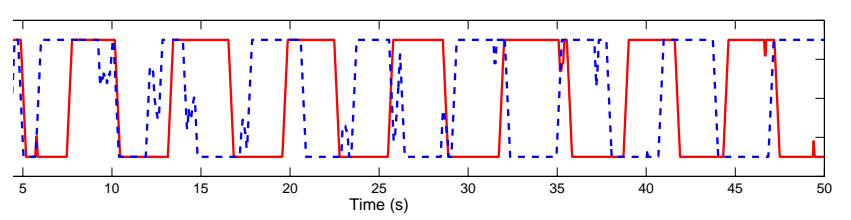

(b) Centralized MPC

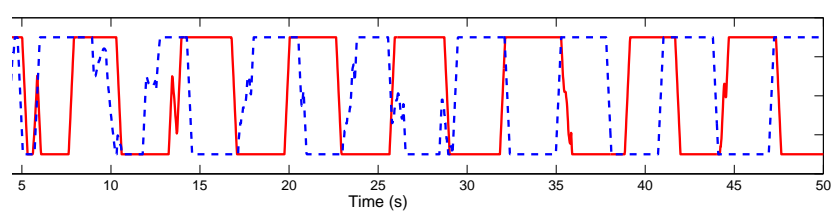

(c) Decentralized MPC

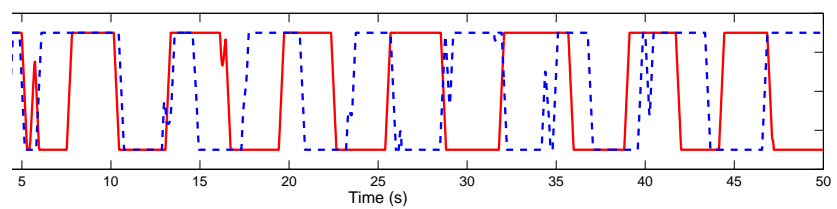

(d) Decentralized MPC (Independent WECs)

Figure 2: The control inputs as a function of time: the solid and dashed lines correspond to the control input on the first and second WEC respectively. The inputs generated by all the candidate controllers satisfy the input constraint. 


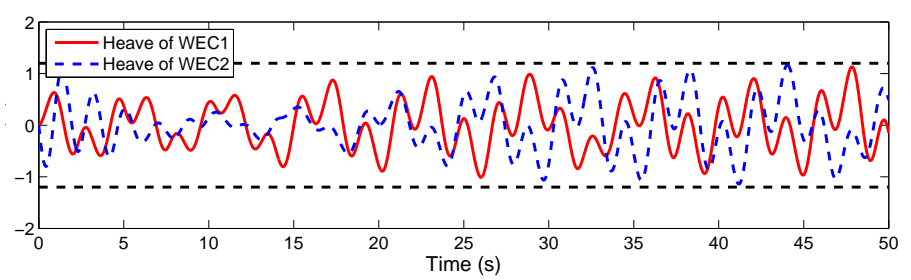

(a) Distributed MPC

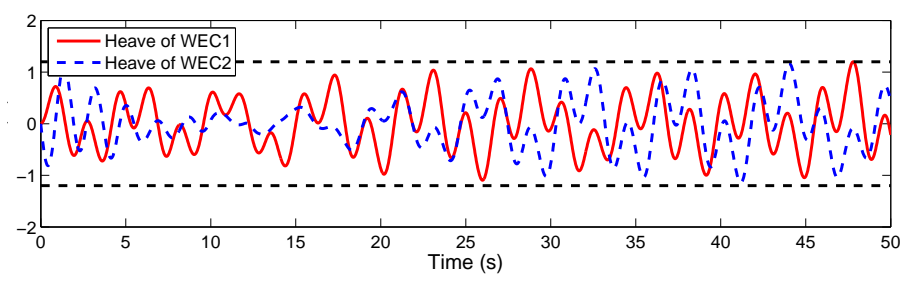

(b) Centralized MPC

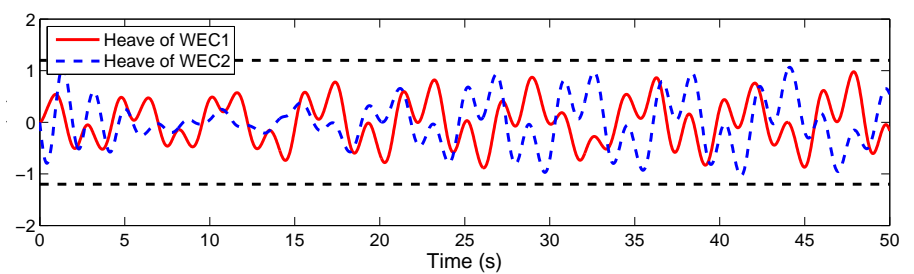

(c) Decentralized MPC

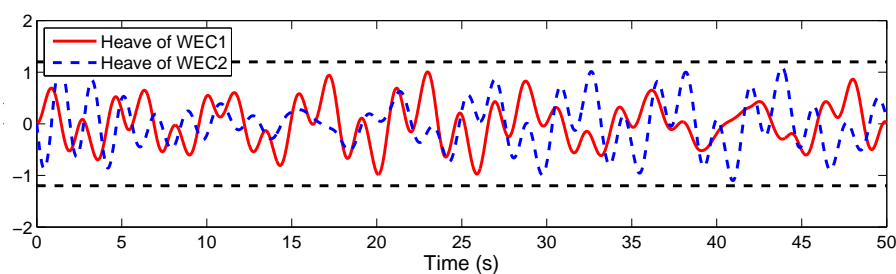

(d) Decentralized MPC (Independent WECs)

Figure 3: The vertical displacement difference between the water level and the mid-point of the float: the solid and dashed lines correspond to the control input on the first and second WEC respectively. The state constraints are satisfied for all the MPC controllers. 


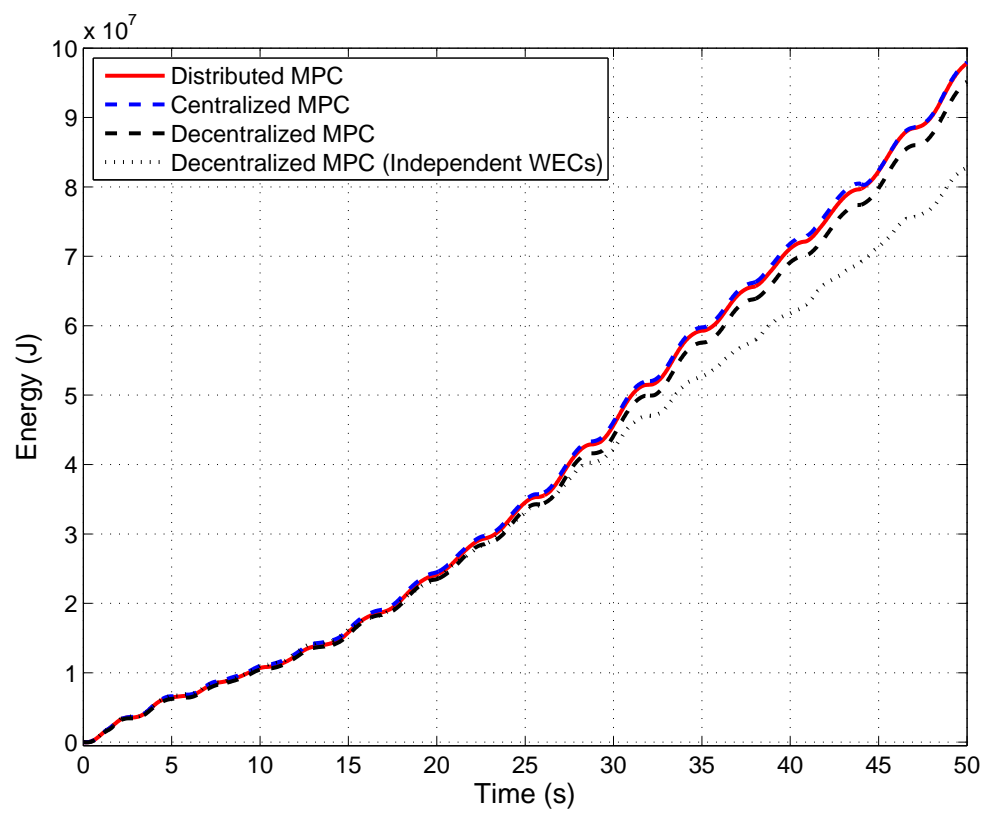

Figure 4: Extracted energy over time. The energy output of the WECs controlled by the distributed MPC is very close to that of WECs controlled by centralized MPC. The WECs controlled by the decentralized MPC generates less energy than the WECs controlled by distributed MPC and centralized MPC. When the two WECs are not coupled by the waves generated by themselves, the two WECs controlled by the decentralized MPC generate the least energy. 
with $E_{a}:=E_{1,2}=E_{1,3}=E_{1,4}, F_{a}:=F_{1,2}=F_{1,3}=F_{1,4}$, and $M_{a}:=$ $M_{1,2}=M_{1,3}=M_{1,4}$.

Type II Since WEC 1 has the same distance $\sqrt{3} d$ to WECs 5 and 6 , we assume $H_{1,5}=H_{1,6}:=H_{b}$. The internal wave generated by WEC 5 and 6 at WEC 1 is $w_{1, b}:=w_{1,5}+w_{1,6}$. Then we have the relation

$$
W_{1, b}(j \omega)=H_{b}(j \omega)\left(Y_{5}(j \omega)+Y_{6}(j \omega)\right)
$$

The state space realization of $H_{b}(j \omega)$ is

$$
\begin{aligned}
\xi_{1, b}(k+1) & =E_{b} \xi_{1, b}(k)+F_{b}\left(y_{5}(k)+y_{6}(k)\right) \\
w_{1, b}(k) & =M_{b} \xi_{1, b}(k)
\end{aligned}
$$

with $E_{b}:=E_{1,5}=E_{1,6}, F_{b}:=F_{1,5}=F_{1,6}$, and $M_{b}:=M_{1,5}=M_{1,6}$.

Type III WEC 1 has a distance $2 d$ to WEC 7 ; we denote this dynamics as $H_{c}:=H_{1,7}$, whose state space realization is

$$
\begin{aligned}
\xi_{1, c}(k+1) & =E_{c} \xi_{1, c}(k)+F_{c} y_{7}(k) \\
w_{1, c}(k) & =M_{c} \xi_{1, c}(k)
\end{aligned}
$$

with $E_{c}:=E_{1,7}, F_{c}:=F_{1,7}$ and $M_{c}:=M_{1,7}$.

In a similar way, the models of the internal waves acting on the WECs 2, 3, 5, 6 and 7 can also be derived in a similar way. Define the state of each WEC as $\mathbf{x}_{i}:=\left[x_{i}^{T}, \xi_{i, a}^{T}, \xi_{i, b}^{T}, \xi_{i, c}^{T}\right]^{T}$, then by applying (1) the partitioned models for these WECs are

$$
\begin{aligned}
{\left[\begin{array}{c}
x_{i}(k+1) \\
\xi_{i, a}(k+1) \\
\xi_{i, b}(k+1) \\
\xi_{i, c}(k+1)
\end{array}\right]=} & {\left[\begin{array}{cccc}
A & B_{w} M_{a} & B_{w} M_{b} & B_{w} M_{c} \\
0 & E_{a} & 0 & 0 \\
0 & 0 & E_{b} & 0 \\
0 & 0 & 0 & E_{c}
\end{array}\right]\left[\begin{array}{c}
x_{i}(k) \\
\xi_{i, a}(k) \\
\xi_{i, b}(k) \\
\xi_{i, c}(k)
\end{array}\right]+\left[\begin{array}{c}
B_{u} \\
0 \\
0 \\
0
\end{array}\right] u_{i}(k) } \\
& +\left[\begin{array}{c}
B_{w} \\
0 \\
0 \\
0
\end{array}\right] w_{f, i}(k)+\sum_{j \in \mathbb{J}_{i}}\left[\begin{array}{cccc}
0 & 0 & 0 & 0 \\
F_{a} C & 0 & 0 & 0 \\
0 & 0 & 0 & 0 \\
0 & 0 & 0 & 0
\end{array}\right]\left[\begin{array}{c}
x_{j}(k) \\
\xi_{j, a}(k) \\
\xi_{j, b}(k) \\
\xi_{j, c}(k)
\end{array}\right] \\
& +\sum_{l \in \mathbb{L}_{i}}\left[\begin{array}{cccc}
0 & 0 & 0 & 0 \\
0 & 0 & 0 & 0 \\
F_{c} C & 0 & 0 & 0 \\
0 & 0 & 0 & 0
\end{array}\right]\left[\begin{array}{c}
x_{l}(k) \\
\xi_{l, a}(k) \\
\xi_{l, b}(k) \\
\xi_{l, c}(k)
\end{array}\right]+\sum_{t \in \mathbb{T}_{i}}\left[\begin{array}{cccc}
0 & 0 & 0 & 0 \\
0 & 0 & 0 & 0 \\
0 & 0 & 0 & 0 \\
F_{c} C & 0 & 0 & 0
\end{array}\right]\left[\begin{array}{c}
x_{t}(k) \\
\xi_{t, a}(k) \\
\xi_{t, b}(k) \\
\xi_{t, c}(k)
\end{array}\right]
\end{aligned}
$$




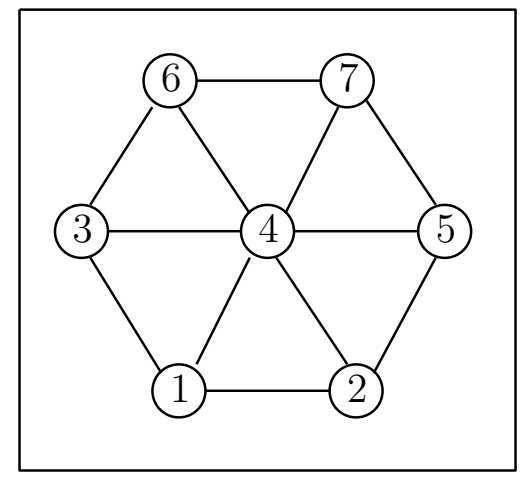

Figure A.5: An array of 7 WECs

where $i=2,3,5,6,7 ; \mathbb{J}_{1}=\{2,3,4\}, \mathbb{L}_{1}=\{5,6\}, \mathbb{T}_{1}=\{7\} ; \mathbb{J}_{2}=\{1,4,5\}$, $\mathbb{L}_{2}=\{3,7\}, \mathbb{T}_{2}=\{6\} ; \mathbb{J}_{3}=\{1,4,6\}, \mathbb{L}_{3}=\{2,7\}, \mathbb{T}_{3}=\{5\} ; \mathbb{J}_{5}=\{2,4,7\}$, $\mathbb{L}_{5}=\{1,6\}, \mathbb{T}_{5}=\{3\} ; \mathbb{J}_{6}=\{3,4,7\}, \mathbb{L}_{6}=\{1,5\}, \mathbb{T}_{6}=\{2\} ; \mathbb{J}_{7}=\{4,5,6\}$, $\mathbb{L}_{7}=\{2,3\}, \mathbb{T}_{7}=\{1\}$.

Finally, the model for WEC 4 is

$$
\begin{aligned}
{\left[\begin{array}{c}
x_{4}(k+1) \\
\xi_{4, a}(k+1) \\
\xi_{4, b}(k+1) \\
\xi_{4, c}(k+1)
\end{array}\right]=} & {\left[\begin{array}{cccc}
A & B_{w} M_{a} & 0 & 0 \\
0 & E_{a} & 0 & 0 \\
0 & 0 & 0 & 0 \\
0 & 0 & 0 & 0
\end{array}\right]\left[\begin{array}{c}
x_{4}(k) \\
\xi_{4, a}(k) \\
\xi_{4, b}(k) \\
\xi_{4, c}(k)
\end{array}\right]+\left[\begin{array}{c}
B_{u} \\
0 \\
0 \\
0
\end{array}\right] u_{4}(k) } \\
& +\left[\begin{array}{c}
B_{w} \\
0 \\
0 \\
0
\end{array}\right] w_{f, 4}(k)+\sum_{j \in \mathbb{J}_{4}}\left[\begin{array}{cccc}
0 & 0 & 0 & 0 \\
F_{a} C & 0 & 0 & 0 \\
0 & 0 & 0 & 0 \\
0 & 0 & 0 & 0
\end{array}\right]\left[\begin{array}{c}
x_{j}(k) \\
\xi_{j, a}(k) \\
\xi_{j, b}(k) \\
\xi_{j, c}(k)
\end{array}\right]
\end{aligned}
$$

with $\mathbb{J}_{4}=\{1,2,3,5,6,7\}$. Here $\xi_{4, a}:=\xi_{4,1}+\xi_{4,2}+\xi_{4,3}+\xi_{4,5}+\xi_{4,6}+\xi_{4,7}$, and $\xi_{4, b}$ and $\xi_{4, c}$ are dummy variables used to augment the state variable $\mathbf{x}_{4}$ to the same dimension with those of other WECs' state variables.

Based on the models of each WEC, a model for the dynamics including all the 7 WECs and the internal wave models can be established by straightforward manipulations; the details of this is skipped here due to the space limit. 
Appendix B. Derivation of (23) and (24) in Section 4

Propagating model (10) through control horizon $N$ gives

$$
\overline{\mathbf{x}}_{i}=\Lambda_{x, i} \mathbf{x}_{i}+\Phi_{u, i} \overline{\mathbf{u}}_{i}+\Phi_{w, i} \overline{\mathbf{w}}_{f, i}+\sum_{j \neq i}^{m} \Phi_{i, j} \overline{\mathbf{x}}_{j}
$$

with $i=1, \ldots, m$ and

$$
\begin{aligned}
& \Lambda_{x, i}=\left[\begin{array}{c}
I \\
\mathbf{A}_{i} \\
\mathbf{A}_{i}^{2} \\
\vdots \\
\mathbf{A}_{i}^{N}
\end{array}\right] \quad \Phi_{u, i}=\left[\begin{array}{ccccc}
0 & \cdots & \cdots & \cdots & 0 \\
\mathbf{B}_{u, i} & 0 & \cdots & \cdots & 0 \\
\mathbf{A}_{i} \mathbf{B}_{u, i} & \mathbf{B}_{u, i} & 0 & \cdots & 0 \\
\vdots & \vdots & \ddots & \ddots & \vdots \\
\mathbf{A}_{i}^{N-1} \mathbf{B}_{u, i} & \mathbf{A}_{i}^{N-2} \mathbf{B}_{u, i} & \cdots & \mathbf{B}_{u, i} & 0
\end{array}\right] \\
& \Phi_{w, i}=\left[\begin{array}{cccc}
0 & \cdots & \cdots & 0 \\
\mathbf{B}_{w, i} & 0 & \cdots & 0 \\
\mathbf{A}_{i} \mathbf{B}_{w, i} & \mathbf{B}_{w, i} & 0 & \cdots \\
\vdots & \vdots & \ddots & \ddots \\
\mathbf{A}_{i}^{N-1} \mathbf{B}_{w, i} & \mathbf{A}_{i}^{N-2} \mathbf{B}_{w, i} & \cdots & \mathbf{B}_{w, i}
\end{array}\right] \\
& \Phi_{i, j}=\left[\begin{array}{ccccc}
0 & \cdots & \cdots & \cdots & 0 \\
\mathbf{F}_{i, j} & 0 & \cdots & \cdots & 0 \\
\mathbf{A}_{i} F_{i, j} & \mathbf{F}_{i, j} & 0 & \cdots & 0 \\
\vdots & \vdots & \ddots & \ddots & \vdots \\
\mathbf{A}_{i}^{N-2} \mathbf{F}_{i, j} & \mathbf{A}_{i}^{N-3} \mathbf{F}_{i, j} & \cdots & \mathbf{F}_{i, j} & 0
\end{array}\right]
\end{aligned}
$$

From equation (B.1) with $i=1, \ldots, m$, we can derive

$$
\Phi_{x} \overline{\mathbf{x}}=\Phi_{u} \overline{\mathbf{u}}+\Lambda \mathbf{x}+\Phi_{w} \overline{\mathbf{w}}
$$

with

$$
\overline{\mathbf{x}}=\left[\begin{array}{c}
\overline{\mathbf{x}}_{1} \\
\overline{\mathbf{x}}_{2} \\
\vdots \\
\overline{\mathbf{x}}_{m}
\end{array}\right] \quad \overline{\mathbf{u}}=\left[\begin{array}{c}
\overline{\mathbf{u}}_{1} \\
\overline{\mathbf{u}}_{2} \\
\vdots \\
\overline{\mathbf{u}}_{m}
\end{array}\right]
$$


and

$$
\boldsymbol{\Phi}_{x}=\left[\begin{array}{cccc}
I & -\Phi_{1,2} & \cdots & -\Phi_{1, m} \\
-\Phi_{2,1} & I & \cdots & -\Phi_{2, m} \\
\vdots & \vdots & \ddots & \vdots \\
-\Phi_{m, 1} & -\Phi_{m, 2} & \cdots & I
\end{array}\right]
$$

$\boldsymbol{\Phi}_{u}=\operatorname{blkdiag}\left(\Phi_{u, 1}, \Phi_{u, 2}, \ldots, \Phi_{u, m}\right), \boldsymbol{\Phi}_{w}=\operatorname{blkdiag}\left(\Phi_{w, 1}, \Phi_{w, 2}, \ldots, \Phi_{w, m}\right)$ and $\boldsymbol{\Lambda}=\operatorname{blkdiag}\left(\Lambda_{x, 1}, \Lambda_{x, 2}, \ldots, \Lambda_{x, m}\right)$.

Since $\boldsymbol{\Phi}_{x}$ is invertible, pre-multiplying $\boldsymbol{\Phi}_{x}^{-1}$ on both sides of the equation (B.2) gives

$$
\overline{\mathbf{x}}=\boldsymbol{\Phi}_{x}^{-1} \boldsymbol{\Phi}_{u} \overline{\mathbf{u}}+\boldsymbol{\Phi}_{x}^{-1} \boldsymbol{\Lambda} \mathbf{x}+\boldsymbol{\Phi}_{x}^{-1} \boldsymbol{\Phi}_{w} \overline{\mathbf{w}}
$$

Moreover, we have

$$
\overline{\mathbf{u}}=\mathbf{T}_{\mathbf{u}} \mathbf{u}(k-1)+\mathbf{T}_{\Delta} \Delta \overline{\mathbf{u}}
$$

with $\mathbf{T}_{\mathbf{u}}=\operatorname{blkdiag}\left(T_{u}, \cdots, T_{u}\right), \mathbf{T}_{\boldsymbol{\Delta}}=\operatorname{blkdiag}\left(T_{\Delta}, \cdots, T_{\Delta}\right)$.

$$
\overline{\mathbf{u}}=\left[\begin{array}{c}
\overline{\mathbf{u}}_{1} \\
\overline{\mathbf{u}}_{2} \\
\vdots \\
\overline{\mathbf{u}}_{\mathrm{m}}
\end{array}\right], \quad \Delta \overline{\mathbf{u}}=\left[\begin{array}{c}
\Delta \overline{\mathbf{u}}_{1} \\
\Delta \overline{\mathbf{u}}_{2} \\
\vdots \\
\Delta \overline{\mathbf{u}}_{\mathrm{m}}
\end{array}\right]
$$

and $\boldsymbol{\Delta} \overline{\mathbf{u}}_{\mathbf{i}}:=\left[\Delta u_{i}(k), \cdots, \Delta u_{i}(k+M-1)\right]^{T}$ is the input slew rate trajectory for WEC $i$.

Substituting (B.4) into (B.3) leads to

$$
\overline{\mathbf{x}}=\boldsymbol{\Phi}_{x}^{-1} \boldsymbol{\Phi}_{u} \mathbf{T}_{u} \mathbf{u}(k-1)+\boldsymbol{\Phi}_{x}^{-1} \boldsymbol{\Phi}_{u} \mathbf{T}_{\Delta} \Delta \overline{\mathbf{u}}+\boldsymbol{\Phi}_{x}^{-1} \boldsymbol{\Lambda} \mathbf{x}+\boldsymbol{\Phi}_{x}^{-1} \boldsymbol{\Phi}_{w} \overline{\mathbf{w}}
$$

Define $\overline{\mathbf{C}}:=\operatorname{blkdiag}\left(\overline{\mathbf{C}}_{1}, \ldots, \overline{\mathbf{C}}_{m}\right)$ with $\overline{\mathbf{C}}_{i}:=\operatorname{blkdiag}(\underbrace{\mathbf{C}_{i}, \ldots, \mathbf{C}_{i}}_{N+1})$ so that $\overline{\mathbf{y}}=\overline{\mathbf{C}} \overline{\mathbf{x}}$. Then pre-multiplying $\overline{\mathbf{C}}$ on both sides of the equation (B.3) gives (21), with $\boldsymbol{\Theta}_{u}:=\overline{\mathbf{C}} \boldsymbol{\Phi}_{x}^{-1} \boldsymbol{\Phi}_{u} \mathbf{T}_{u}, \boldsymbol{\Psi}:=\overline{\mathbf{C}} \boldsymbol{\Phi}_{x}^{-1} \boldsymbol{\Phi}_{u} \mathbf{T}_{\Delta}, \boldsymbol{\Theta}_{x}:=\overline{\mathbf{C}} \boldsymbol{\Phi}_{x}^{-1} \boldsymbol{\Lambda}$ and $\boldsymbol{\Theta}_{w}:=$ $\overline{\mathbf{C}} \boldsymbol{\Phi}_{x}^{-1} \boldsymbol{\Phi}_{w}$.

By defining $\overline{\mathbf{C}}_{z}$ in a similar way to extract the state subject to constraint, we have (22), with $\boldsymbol{\Theta}_{z, u}:=\overline{\mathbf{C}}_{z} \boldsymbol{\Phi}_{x}^{-1} \boldsymbol{\Phi}_{u} \mathbf{T}_{u}, \boldsymbol{\Psi}_{z}:=\overline{\mathbf{C}}_{z} \boldsymbol{\Phi}_{x}^{-1} \boldsymbol{\Phi}_{u} \mathbf{T}_{\Delta}, \boldsymbol{\Theta}_{z, x}:=$ $\overline{\mathbf{C}}_{z} \boldsymbol{\Phi}_{x}^{-1} \boldsymbol{\Lambda}, \boldsymbol{\Theta}_{z, w}:=\overline{\mathbf{C}}_{z} \boldsymbol{\Phi}_{x}^{-1} \boldsymbol{\Phi}_{w}$.

(23) and (24) and be derived straightforwardly from (21) and (22). 


\section{Bibliography}

[1] G. Li, M. B. Belmont, Model predictive control of sea wave energy converters - Part I: the case of a single device, submitted to Renewable Energy (2013).

[2] E. Camponogara, D. Jia, B. Krogh, S. Talukdar, Distributed model predictive control, IEEE Control Systems Magazine 22 (1) (2002) 44 -52 .

[3] A. Venkat, I. Hiskens, J. Rawlings, S. Wright, Distributed mpc strategies with application to power system automatic generation control, IEEE Transactions on Control Systems Technology 16 (6) (2008) $1192-1206$.

[4] E. L. Morris, H. K. Zienkiewicz, M. R. Belmont, Short term forecasting of the sea surface shape, Int. J. Shipbld. Prog. 45 (1998) 381-400.

[5] M. R. Belmont, J. M. K. Horwood, R. W. F. Thurley, J. Baker, Filters for linear sea-wave prediction, Ocean Engineering 33 (2006) 2332-2351.

[6] M. R. Belmont, J. M. K. Horwood, The effect of frequency distribution in simulations of deterministic sea wave prediction, Int. Shipbld. Prog. (46) (1998) 265-276.

[7] M. R. Belmont, E. L. Morris, Adaptive measurement and signal processing strategies associated with deterministic sea wave prediction, Cambridge, UK, 1994, pp. 21-26.

[8] D. R. Edgar, J. M. K. Horwood, R. Thurley, M. R. Belmont, The effects of parameters on the maximum prediction time possible in short term forecasting of the sea surface shape, Int. Shipbuild. Progr. 47 (251) (2000) 287-301.

[9] L. Abusedra, M. R. Belmont, Prediction diagrams for deterministic sea wave prediction and the introduction of the data extension prediction method, Int. J. Sipbuilding Prog. 48 (1) (2011) 59-81.

[10] P. Naaijen, R. Huijsmans, Real time wave forecasting for real time ship motion predictions, in: 27th International Conference on Offshore Mechanics and Arctic Engineering (OMAE), Portugal, 2008, pp. 377-387. 
[11] J. Falnes, Radiation impedance matrix and optimum power absorption for interacting oscillators in surface waves, Applied ocean research 2 (2) (1979) 75-80.

[12] G. Li, G. Weiss, M. Mueller, S. Townley, M. B. Belmont, Wave energy converter control by wave prediction and dynamic programming, Renewable Energy 48 (2012) 392-403.

[13] G. Weiss, G. Li, M. Mueller, S. Townley, , M. R. Belmont, Optimal control of wave energy converters using deterministic sea wave prediction, in: The Energy \& Materials Research Conference, Torremolinos, Malaga, Spain, 2012.

[14] J. Falnes, Ocean Waves and Oscillating Systems, Cambridge University Press, 2002. 